\title{
On an Initial Boundary Value Problem for a Class of Odd Higher Order Pseudohyperbolic Integrodifferential Equations
}

\author{
Said Mesloub \\ Department of Mathematics, College of Sciences, King Saud University, P.O. Box 2455, Riyadh 11451, Saudi Arabia \\ Correspondence should be addressed to Said Mesloub; mesloubs@yahoo.com
}

Received 2 March 2014; Accepted 30 May 2014; Published 15 June 2014

Academic Editor: Yansheng Liu

Copyright ( 2014 Said Mesloub. This is an open access article distributed under the Creative Commons Attribution License, which permits unrestricted use, distribution, and reproduction in any medium, provided the original work is properly cited.

\begin{abstract}
This paper is devoted to the study of the well-posedness of an initial boundary value problem for an odd higher order nonlinear pseudohyperbolic integrodifferential partial differential equation. We associate to the equation $n$ nonlocal conditions and $n+1$ classical conditions. Upon some a priori estimates and density arguments, we first establish the existence and uniqueness of the strongly generalized solution in a class of a certain type of Sobolev spaces for the associated linear mixed problem. On the basis of the obtained results for the linear problem, we apply an iterative process in order to establish the well-posedness of the nonlinear problem.
\end{abstract}

\section{Introduction}

Classical and nonclassical and local and nonlocal initial boundary value problems for partial differential equations are widely studied and athre being studied nowadays. One of the most important and crucial tools to be applied to partial differential equations is functional analysis. It is the universal language of mathematics. No serious study in partial differential equations, mathematical physics, numerical analysis, mathematical economics, or control theory is conceivable without a broad solicitation to methods and results of the functional analysis and its applications.

The main objective of this research work is to develop one of the powerful methods of functional analysis, namely, the energy inequality method for a certain classes of partial differential equations with nonlocal constraints of convolution type in some functional spaces of Sobolev type. This method, based on the ideas of Petrovski [1], Leray [2], Garding [3], and presented on a method form by Dezin [4], was used to investigate and study different categories of mixed problems related to elliptic, parabolic, and hyperbolic equations [512], mixed equations [13-15], nonclassical equations [16, 17], and operational equations $[18,19]$, with classical conditions of types: Cauchy, Dirichlet, Neumann, and Robinson.

Mixed nonlocal problems are especially inspired from modern physics and technological sciences and they describe many physical and biological phenomena. That is in terms of applications, nonlocal mixed problems are widely applied in medical science, biological processes, chemical reaction diffusion, heat conduction processes, population dynamics, thermoelasticity, control theory, and in so many other domains of research. It is worth to mention that for these types of problems, we cannot measure the data directly on the boundary, but we only know the average value of the solution on the domain.

For second order parabolic equations with nonlocal conditions, the reader should refer to [20-23]. For hyperbolic equations and pseudoparabolic equations with purely or one integral conditions, the reader should refer to [2431]. The reader could also refer to a recent paper dealing with a higher dimension Boussinesq equation with a purely nonlocal condition [32]. This paper is organized as follows. In Section 2, we pose and set the problem to be solved. In Section 3, we give some notations, introduce the functional frame, and state some important inequalities that will be used in the sequel. Section 4 is devoted to the proof of the uniqueness of the solution of the associated linear problem. In Section 5, we establish and prove the existence of solution of the posed associated linear problem. In the last Section, Section 6, we solve the nonlinear problem. On the basis of the results obtained in Sections 4 and 5 , and by using an iterative process, we prove the existence and uniqueness of 
the solution of problem (1)-(6). Some proofs of Sections 3, 4, and 5 are given in Appendices A and B at the end of Section 6. At the end of the paper, we give a set of references.

\section{Problem Setting}

In the rectangle $Q=(0, b) \times(0, T)$, where $0<b<\infty$ and $0<T<\infty$, we consider the nonlinear higher order pseudohyperbolic differential equation of odd order

$$
\begin{aligned}
\frac{\partial^{2} u}{\partial t^{2}} & +(-1)^{m} \alpha(t) \frac{\partial}{\partial t}\left(\frac{\partial^{2 m+1} u}{\partial x^{2 m+1}}\right) \\
& =f\left(x, t, \frac{x^{m-1} * u}{(m-1) !}, \frac{x^{m-1} * \partial u / \partial t}{(m-1) !}\right),
\end{aligned}
$$

where

$$
\frac{x^{m-1} * u(x, t)}{(m-1) !}=\int_{0}^{x} \frac{(x-z)^{m-1} u(z, t)}{(m-1) !} d z .
$$

In (1), $f$ is a given function which will be specified later on and $\alpha(t)$ is a function satisfying the conditions

(H1) $c_{0} \leq \alpha(t)$ for all $t \in[0, T]$,

(H2) $\alpha(t) \leq c_{1}, c_{2} \leq \alpha^{\prime}(t) \leq c_{3}, \alpha^{\prime \prime}(t) \leq c_{4}, 0 \leq \alpha^{\prime \prime \prime}(t)$ for all $t \in[0, T]$ and all constants $c_{i} ; i=\overline{0,4}$ are strictly positive.

To (1), we associate the initial conditions

$$
\begin{aligned}
& \ell_{1} u=u(x, 0)=\varphi_{1}(x), \\
& \ell_{2} u=u_{t}(x, 0)=\varphi_{2}(x),
\end{aligned}
$$

the Dirichlet boundary condition

$$
u(0, t)=0,
$$

the Neumann boundary conditions

$$
\frac{\partial^{j} u(b, t)}{\partial x^{j}}=0, \quad j=\overline{m+1,2 m}
$$

and the nonlocal conditions

$$
\left.\left(x^{k-1} * u(x, t)\right)\right|_{x=b}=0, \quad k=\overline{1, m},
$$

where the data functions $\varphi_{1}$ and $\varphi_{2}$ satisfy the compatibility conditions

$$
\begin{gathered}
\varphi_{1}(0)=\varphi_{2}(0)=0, \\
\frac{\partial^{j} \varphi_{1}(b)}{\partial x^{j}}=\frac{\partial^{j} \varphi_{2}(b)}{\partial x^{j}}=0, \quad j=\overline{m+1,2 m}, \\
\left.\left(x^{k-1} * \varphi_{1}(x)\right)\right|_{x=b}=0,\left.\quad\left(x^{k-1} * \varphi_{2}(x)\right)\right|_{x=b}=0, \\
k=\overline{1, m} .
\end{gathered}
$$

In this paper, we are concerned with the proof of wellposedness of the nonlinear nonlocal initial boundary value problem (1)-(6) in some weighted Sobolev spaces.

The main tools used in our proofs are mainly based on some iterative processes, some priori bounds, and some density arguments.

\section{Functional Framework, Notations, and Some Inequalities}

For the investigation of problem (1)-(6), we need the following function spaces.

Let $L^{2}(Q)$ be the usual Hilbert space of square integrable functions and let $H(0, b)=B_{2}^{m}(0, b)$ [24] be the Hilbert space of Sobolev type constituted of functions $u \in L^{2}(0, b)$ if $m=0$ and of functions $u$ such that $\int_{0}^{x}\left((x-z)^{m-1} u(z) /(m-1) !\right) d z \in$ $L^{2}(0, b)$, if $m \geq 1$, with inner product

$$
\begin{aligned}
(u, v)_{H(0, b)}=\int_{0}^{b} & {\left[\left(\int_{0}^{x} \frac{(x-z)^{m-1} u(z, t)}{(m-1) !} d z\right)\right.} \\
& \left.\times\left(\int_{0}^{x} \frac{(x-z)^{m-1} v(z, t)}{(m-1) !} d z\right)\right] d x
\end{aligned}
$$

and with associated norm

$$
\|u\|_{H(0, b)}=\left\|\int_{0}^{x} \frac{(x-z)^{m-1} u(z, t)}{(m-1) !} d z\right\|_{L^{2}(0, b)} \quad \text { for } m \geq 1 .
$$

Corollary 1. For all $m \in \mathbb{N}^{*}$, one has the inequality

$$
\|u\|_{H(0, b)}^{2} \leq \frac{b^{2}}{2}\left\|\int_{0}^{x} \frac{(x-z)^{m-2} u(z, t)}{(m-2) !} d z\right\|_{L^{2}(0, b)}^{2} .
$$

Proof. See Appendix A.

Corollary 2. For all $m \in I N^{*}$, one has the inequalities

$$
\|u\|_{H(0, b)}^{2} \leq\left(\frac{b^{2}}{2}\right)^{m}\|u\|_{L^{2}(0, b)}^{2}
$$

$$
\left\|\int_{0}^{x} \frac{(x-z)^{2 m-1} u(z, t)}{(2 m-1) !} d z\right\|_{L^{2}(0, b)}^{2} \leq\left(\frac{b^{2}}{2}\right)^{m}\|u\|_{H(0, b)}^{2} .
$$

One denotes by $L^{2}(0, T ; H(0, b))$ the set of all abstract strongly measurable functions $u$ on $[0, T]$ into $H(0, b)$ such that

$$
\|u\|_{L^{2}(0, T ; H(0, b))}^{2}=\int_{0}^{T}\|u(\cdot, t)\|_{H(0, b)}^{2} d t<\infty .
$$

The space $L^{2}(0, T ; H(0, b))$ is a Hilbert space having the inner product

$$
(u, v)_{L^{2}(0, T ; H(0, b))}=\int_{0}^{T}(u, v)_{H(0, b)} d t .
$$

One can write problem (1)-(6) in an operator form $L u=$ $\mathscr{F}=\left(f, \varphi_{1}, \varphi_{2}\right)$, where $L=\left(\mathfrak{R}, \ell_{1}, \ell_{2}\right)$ is an unbounded operator with domain $D(L)$, acting from a Banach space $E$ into a Hilbert space $F$ constructed as below. One defines the domain of the operator $L$ as the set

$$
D(L)=\left\{\begin{array}{l}
u \in L^{2}(0, T ; H(0, b)), \\
\text { for which } \frac{\partial u}{\partial t}, \frac{\partial^{2} u}{\partial t^{2}}, \frac{\partial^{2} u}{\partial t \partial x}, \ldots, \frac{\partial^{2(m+1)} u}{\partial t \partial x^{2 m+1}} \\
\text { belong to } L^{2}(0, T ; H(0, b)) \\
\text { and conditions (4)-(6) are satisfied. }
\end{array}\right.
$$


The space $E$ is the Banach space of functions $u \in$ $L^{2}(0, T ; H(0, b))$ verifying conditions $(4)-(6)$ and having the norm

$$
\begin{aligned}
\|u\|_{E}^{2} & =\sup _{0 \leq \tau \leq T}\left(\|u(x, \tau)\|_{H(0, b)}^{2}+\left\|u_{t}(x, \tau)\right\|_{H(0, b)}^{2}\right) \\
& =\|u(x, \tau)\|_{H^{1}(0, T ; H(0, b))}^{2} .
\end{aligned}
$$

The space $F$ is the Hilbert $L^{2}(Q) \times H(0, b) \times H(0, b)$ of multivalued functions $\mathscr{F}=\left(f, \varphi_{1}, \varphi_{2}\right)$ with finite norm

$$
\|\mathscr{F}\|_{F}^{2}=\|f\|_{L^{2}(Q)}^{2}+\left\|\varphi_{1}\right\|_{H(0, b)}^{2}+\left\|\varphi_{2}\right\|_{H(0, b)}^{2} .
$$

\section{Uniqueness of Solution of the Associated Linear Problem}

We first treat the following associated linear problem:

$$
\begin{gathered}
\mathfrak{Q} u=u_{t t}+(-1)^{m} \alpha(t) \frac{\partial}{\partial t}\left(\frac{\partial^{2 m+1} u}{\partial x^{2 m+1}}\right)=f(x, t), \\
\ell_{1} u=u(x, 0)=\varphi_{1}(x), \quad \ell_{2} u=u_{t}(x, 0)=\varphi_{2}(x), \\
u(0, t)=0, \quad \frac{\partial^{j} u(b, t)}{\partial x^{j}}=0, \quad j=\overline{m+1,2 m}, \\
\left.\left(x^{k-1} * u(x, t)\right)\right|_{x=b}=0, \quad k=\overline{1, m},
\end{gathered}
$$

where $f\left(x, t,\left(x^{m-1} * u\right) /(m-1) !,\left(x^{m-1} * \partial u / \partial t\right) /(m-1) !\right)$ is replaced by $f(x, t)$.

We establish a priori bound from which we deduce the uniqueness of solution of problem (17).

Theorem 3. If the coefficients $\alpha(t)$ satisfy condition (H1), then there exists a positive constant $M$ independent of $u$ such that

$$
\begin{aligned}
& \|u(x, \tau)\|_{H^{1}(0, T ; H(0, b))}^{2} \\
& \quad \leq M\left(\|f\|_{L^{2}(\mathrm{Q})}^{2}+\left\|\varphi_{1}\right\|_{H(0, b)}^{2}+\left\|\varphi_{2}\right\|_{H(0, b)}^{2}\right),
\end{aligned}
$$

for all $u \in D(L)$.

Proof. See Appendix B.

Proposition 4. The operator $L: E \rightarrow F$ admits a closure.

Proof. See [26].

We denote by $\bar{L}$ the closure of the operator $L$ and by $D(\bar{L})$ the domain of definition of $\bar{L}$ and define the strong solution of problem (17) as the solution of the operator equation $\bar{L} u=\mathscr{F}$.

Inequality (18) can be extended to

$$
\|u(x, \tau)\|_{H^{1}(0, T ; H(0, b))}^{2} \leq M\|\bar{L} u\|_{F}, \quad \forall u \in D(\bar{L}) .
$$

We can deduce from (19) that the strong solution of problem (17) is unique if it exists and depends continuously on $\mathscr{F}=$ $\left(f, \varphi_{1}, \varphi_{2}\right) \in F$ and that the image $\operatorname{Im}(\bar{L})$ of the operator $\bar{L}$ coincides with the set $\overline{\operatorname{Im}(L)}$.

\section{Solvability of the Associated Linear Problem}

Theorem 5. Assume that conditions $H 1$ and $H 2$ are hold. Then problem (17) admits a unique strong solution satisfying $u \in C(0, T ; H(0, b)), u_{t} \in C(0, T ; H(0, b))$ and $u, u_{t}$ depend continuously on the given data and verify

$$
\begin{gathered}
\|u(x, \tau)\|_{H^{1}(0, T ; H(0, b))}^{2} \leq M\|L u\|_{F}, \\
\left\|u_{t}(x, \tau)\right\|_{H^{1}(0, T ; H(0, b))}^{2} \leq M\|L u\|_{F} .
\end{gathered}
$$

Proof. Since $\operatorname{Im}(\bar{L}) \subset F$ is closed and $\operatorname{Im}(\bar{L})=\overline{\operatorname{Im}(L)}$, then in order to prove the existence of the strong solution, we have to show that $\overline{\operatorname{Im}(L)}=F$. We first prove it in the following special case:

Theorem 6. If conditions of Theorem 3 are satisfied and for $\Psi \in$ $L^{2}(Q)$, we have

$$
\left(\frac{\partial^{2} u}{\partial t^{2}}+(-1)^{m} \alpha(t) \frac{\partial}{\partial t}\left(\frac{\partial^{2 m+1} u}{\partial x^{2 m+1}}\right), \Psi\right)_{L^{2}(Q)}=0
$$

for all $u \in D_{0}(L)=\left\{u / u \in D(L), \varphi_{1}(x)=\varphi_{2}(x)=0\right\}$, then $\Psi$ vanishes almost everywhere in $Q$.

Proof. We first define the function $V(x, t)$ by the relation

$$
\begin{aligned}
& V(x, t)-\int_{t}^{T} \Psi(x, \tau) d \tau \\
& \quad=\int_{t}^{T} \frac{\partial}{\partial s}\left(\frac{\partial \alpha(x, s)}{\partial s} \cdot \frac{x^{2 m-1} * u_{t}(x, s)}{(2 m-1) !}\right) d s .
\end{aligned}
$$

We now consider the equation

$$
\alpha(t) \frac{\partial^{2}}{\partial t^{2}}\left(\frac{x^{2 m-1} * u(x, t)}{(2 m-1) !}\right)=V(x, t),
$$

and define $u$ by

$$
u= \begin{cases}0, & 0 \leq t \leq s \\ \int_{t}^{T}(t-\tau) u_{\tau \tau} d \tau, & s \leq t \leq T\end{cases}
$$

Relations (23) and (24) imply that $u$ is in $D_{s}(L) \subseteq D_{0}(L)$, where $D_{s}(L)=\{u / u \in D(L), u=0$ for $t \leq s\}$.

We now have

$$
\Psi(x, t)=-\frac{\partial^{2}}{\partial t^{2}}\left(\alpha(t) \frac{x^{2 m-1} * u_{t}(x, t)}{(2 m-1) !}\right) .
$$


The following lemma shows that $\Psi(x, t)$ given by $(25)$ is in $L^{2}\left(Q_{s}\right)$, where $Q_{s}=(0, b) \times(s, T)$.

Lemma 7. If conditions of Theorem 6 are satisfied, then the function $u$ defined by the relations (23) and (24) has $t$ derivatives up to third order which included are in $L^{2}\left(Q_{s}\right)$.

\section{Proof. See Appendix B.}

We now continue to prove Theorem 6 . We replace $\Psi$ given by (25) in (21) to get

$$
\begin{aligned}
& 2(-1)^{m+1}\left(u_{t t},\left(\alpha(t) \frac{x^{2 m-1} * u_{t}(x, t)}{(2 m-1) !}\right)_{t t}\right)_{L^{2}(\mathrm{Q})} \\
& -2\left(\alpha(t) \frac{\partial^{2 m+1} u_{t}}{\partial x^{2 m+1}},\left(\alpha(t) \frac{x^{2 m-1} * u_{t}(x, t)}{(2 m-1) !}\right)_{t t}\right)_{L^{2}(\mathrm{Q})}=0 .
\end{aligned}
$$

Straight forward successive integration by parts of the two terms in (26) gives

$$
\begin{aligned}
& 2(-1)^{m+1}\left(u_{t t},\left(\alpha(t) \frac{x^{2 m-1} * u_{t}(x, t)}{(2 m-1) !}\right)_{t t}\right)_{L^{2}(\mathrm{Q})} \\
& =\int_{0}^{b} \alpha(s)\left(\frac{x^{m-1} * u_{t t}(x, s)}{(m-1) !}\right)^{2} d x \\
& \quad-\int_{0}^{b} \alpha^{\prime \prime}(T)\left(\frac{x^{m-1} * u_{t}(x, T)}{(m-1) !}\right)^{2} d x \\
& \quad+\int_{Q_{s}} \alpha^{\prime \prime \prime}(t)\left(\frac{x^{m-1} * u_{t}(x, t)}{(m-1) !}\right)^{2} d x d t \\
& \quad-3 \int_{Q_{s}} \alpha^{\prime}(t)\left(\frac{x^{m-1} * u_{t t}(x, t)}{(m-1) !}\right)^{2} d x d t, \\
& -2\left(\alpha(t) \frac{\partial^{2 m+1} u_{t}}{\partial x^{2 m+1}},\left(\alpha(t) \frac{x^{2 m-1} * u_{t}(x, t)}{(2 m-1) !}\right)_{t t}\right)_{L^{2}(\mathrm{Q})} \\
& =\int_{s}^{T} \alpha^{2}(t) u_{t t}^{2}(b, t) d t-\int_{s}^{T} \alpha(t) \alpha^{\prime \prime}(t) u_{t}^{2}(b, t) d t .
\end{aligned}
$$

Substitution of (27) into (26) yields

$$
\begin{aligned}
\int_{0}^{b} \alpha(s) & \left(\frac{x^{m-1} * u_{t t}(x, s)}{(m-1) !}\right)^{2} d x \\
& +\int_{Q_{s}} \alpha^{\prime \prime \prime}(t)\left(\frac{x^{m-1} * u_{t}(x, t)}{(m-1) !}\right)^{2} d x d t \\
& +\int_{s}^{T} \alpha^{2}(t)\left(u_{t t}\right)^{2}(b, t) d t \\
= & \int_{0}^{b} \alpha^{\prime \prime}(T)\left(\frac{x^{m-1} * u_{t}(x, T)}{(m-1) !}\right)^{2} d x
\end{aligned}
$$

$$
\begin{aligned}
& +3 \int_{\mathrm{Q}_{s}} \alpha^{\prime}(t)\left(\frac{x^{m-1} * u_{t t}(x, t)}{(m-1) !}\right)^{2} d x d t \\
& +\int_{s}^{T} \alpha(t) \alpha^{\prime \prime}(t)\left(u_{t}\right)^{2}(b, t) d t .
\end{aligned}
$$

By dropping the second term on the left-hand side (28) and by using conditions $\mathrm{H} 1$ and $\mathrm{H} 2$, we obtain

$$
\begin{gathered}
c_{0} \int_{0}^{b}\left(\frac{x^{m-1} * u_{t t}(x, s)}{(m-1) !}\right)^{2} d x+c_{0}^{2} \int_{s}^{T} u_{t t}^{2}(b, t) d t \\
\leq c_{4} \int_{0}^{b}\left(\frac{x^{m-1} * u_{t}(x, T)}{(m-1) !}\right)^{2} d x \\
\quad+3 c_{3} \int_{Q_{s}}\left(\frac{x^{m-1} * u_{t t}(x, t)}{(m-1) !}\right)^{2} d x d t \\
\quad+c_{1} c_{4} \int_{s}^{T} u_{t}^{2}(b, t) d t .
\end{gathered}
$$

We now consider the two elementary inequalities

$$
\begin{gathered}
2 c_{4} \int_{0}^{b}\left(\frac{x^{m-1} * u_{t}(x, T)}{(m-1) !}\right)^{2} d x \\
\leq 2 c_{4} \int_{Q_{s}}\left(\frac{x^{m-1} * u_{t}(x, t)}{(m-1) !}\right)^{2} d x d t \\
\quad+2 c_{4} \int_{Q_{s}}\left(\frac{x^{m-1} * u_{t t}(x, t)}{(m-1) !}\right)^{2} d x d t, \\
c_{0}^{2} u_{t}^{2}(b, T) \leq c_{0}^{2} \int_{s}^{T} u_{t}^{2}(b, t) d t+c_{0}^{2} \int_{s}^{T} u_{t t}^{2}(b, t) d t .
\end{gathered}
$$

Combination of inequalities (29)-(30) leads to

$$
\begin{gathered}
\int_{0}^{b}\left(\frac{x^{m-1} * u_{t t}(x, s)}{(m-1) !}\right)^{2} d x \\
+\int_{0}^{b}\left(\frac{x^{m-1} * u_{t}(x, T)}{(m-1) !}\right)^{2} d x+u_{t}^{2}(b, T) \\
\leq c_{7}\left(\int_{Q_{s}}\left(\frac{x^{m-1} * u_{t t}(x, t)}{(m-1) !}\right)^{2} d x d t\right. \\
+\int_{Q_{s}}\left(\frac{x^{m-1} * u_{t}(x, t)}{(m-1) !}\right)^{2} d x d t \\
\left.+\int_{s}^{T} u_{t}^{2}(b, t) d t\right)
\end{gathered}
$$

where

$$
\mathcal{c}_{7}=\frac{\max \left(c_{0}^{2}+c_{1} c_{4}, 3 c_{3}+2 c_{4}\right)}{\min \left(c_{0}, c_{0}^{2}, c_{4}\right)} .
$$


We now introduce a new function $\beta$ defined by $\beta(x, t)=$ $\int_{t}^{T} u_{\tau \tau} d \tau$, then $\partial u(x, t) / \partial t=\beta(x, s)-\beta(x, t)$, and $\partial u(x, T) / \partial t=\beta(x, s)$, and we have

$$
\begin{gathered}
\int_{0}^{b}\left(\frac{x^{m-1} * u_{t t}(x, s)}{(m-1) !}\right)^{2} d x+\left(1-2 c_{7}(T-s)\right) \\
\times\left(\int_{0}^{b}\left(\frac{x^{m-1} * \beta(x, s)}{(m-1) !}\right)^{2} d x+\beta^{2}(b, s)\right) \\
\leq 2 c_{7}\left(\int_{Q_{s}}\left(\frac{x^{m-1} * u_{t t}(x, t)}{(m-1) !}\right)^{2} d x d t\right. \\
+\int_{Q_{s}}\left(\frac{x^{m-1} * \beta(x, t)}{(m-1) !}\right)^{2} d x d t \\
\left.+\int_{s}^{T} \beta^{2}(b, t) d t\right) .
\end{gathered}
$$

If we choose $s_{0}>0$ such that $1-2 c\left(T-s_{0}\right)=1 / 2$, then for all $s \in\left[T-s_{0}, T\right]$, inequality (33) implies that

$$
\begin{gathered}
\int_{0}^{b}\left(\frac{x^{m-1} * u_{t t}(x, s)}{(m-1) !}\right)^{2} d x \\
+\int_{0}^{b}\left(\frac{x^{m-1} * \beta(x, s)}{(m-1) !}\right)^{2} d x+\beta^{2}(b, s) \\
\leq 4 c_{7}\left(\int_{Q_{s}}\left(\frac{x^{m-1} * u_{t t}(x, t)}{(m-1) !}\right)^{2} d x d t\right. \\
+\int_{Q_{s}}\left(\frac{x^{m-1} * \beta(x, t)}{(m-1) !}\right)^{2} d x d t \\
\left.+\int_{s}^{T} \beta^{2}(b, t) d t\right) .
\end{gathered}
$$

Inequality (34) can be written in the form of

$$
-\frac{\partial M}{\partial s} \leq 4 c_{7} M(s)
$$

where

$$
\begin{aligned}
M(x)= & \int_{\mathrm{Q}_{s}}\left(\frac{x^{m-1} * u_{t t}(x, t)}{(m-1) !}\right)^{2} d x d t \\
& +\int_{\mathrm{Q}_{s}}\left(\frac{x^{m-1} * \beta(x, t)}{(m-1) !}\right)^{2} d x d t \\
& +\int_{s}^{T} \beta^{2}(b, t) d t .
\end{aligned}
$$

It follows from (35) that $M(s) \exp \left(4 c_{7} s\right) \leq 0$ from which it follows that $\Psi=0$ almost everywhere in $Q_{T-s_{0}}$. By reiterating the same procedure, we deduce that $\Psi=0$ a.e., in $Q$. We now continue the proof of Theorem 5.
We consider a function $W=\left(\Psi, N_{1}, N_{2}\right)$ in $\operatorname{Im}(L)^{\perp}$. The function $u$ satisfies

$$
\begin{aligned}
(L u, W)_{F}= & (\mathfrak{L u}, \Psi)_{L^{2}(Q)}+\left(\ell_{1} u, N_{1}\right)_{H(0, b)} \\
& +\left(\ell_{2} u, N_{2}\right)_{H(0, b)} \\
= & 0 .
\end{aligned}
$$

If we pick an element $u$ in $D_{0}(L)$, equality (37) becomes

$$
(\mathfrak{Q} u, \Psi)_{L^{2}(Q)}=0, \quad \forall u \in D_{0}(L) .
$$

By virtue of Theorem 6, we deduce that $\Psi=0$, and (37) then takes the form

$$
\left(\ell_{1} u, N_{1}\right)_{H(0, b)}+\left(\ell_{2} u, N_{2}\right)_{H(0, b)}=0, \quad \forall u \in D(L) .
$$

It follows from (39) that $N_{1}=0, N_{2}=0$. This results from the fact that the quantities $\ell_{1} u$ and $\ell_{2} u$ vanish independently and that the set of values of the trace operators $\ell_{1}$ and $\ell_{2}$ is dense in $H(0, b)$.

\section{The Nonlinear Problem}

On the basis of the results obtained for the linear case, we are now able to establish the existence and uniqueness results for the nonlinear problem (1)-(6).

Observe that the function $y=u-Y$ solves the problem

$$
\begin{gathered}
\mathfrak{L} y=y_{t t}+(-1)^{m} \alpha(t) \frac{\partial}{\partial t}\left(\frac{\partial^{2 m+1} y}{\partial x^{2 m+1}}\right) \\
=F\left(x, t, \frac{x^{m-1} * y}{(m-1) !}, \frac{x^{m-1} * y_{t}}{(m-1) !}\right), \\
y(x, 0)=0, \quad y_{t}(x, 0)=0, \\
y(0, t)=0, \quad \frac{\partial^{j} y(b, t)}{\partial x^{j}}=0, \\
\left.\left(x^{k-1} * y(x, t)\right)\right|_{x=b}=0, \\
k=\overline{1, m}, \quad j=\overline{m+1,2 m},
\end{gathered}
$$

where

$$
\begin{aligned}
& F\left(x, t, \frac{x^{m-1} * y}{(m-1) !}, \frac{x^{m-1} * y_{t}}{(m-1) !}\right) \\
& \quad=f\left(x, t, \frac{x^{m-1} *(y+Y)}{(m-1) !}, \frac{x^{m-1} *\left(y_{t}+Y_{t}\right)}{(m-1) !}\right),
\end{aligned}
$$


whenever $u$ and $Y$ are, respectively, solutions of the problems

$$
\begin{gathered}
\mathfrak{Q} u=u_{t t}+(-1)^{m} \alpha(t) \frac{\partial}{\partial t}\left(\frac{\partial^{2 m+1} u}{\partial x^{2 m+1}}\right) \\
=f\left(x, t, \frac{x^{m-1} * u}{(m-1) !}, \frac{x^{m-1} * u_{t}}{(m-1) !}\right), \\
u(x, 0)=\varphi_{1}(x), \quad u_{t}(x, 0)=\varphi_{2}(x), \\
u(0, t)=0, \quad \frac{\partial^{j} u(b, t)}{\partial x^{j}}=0, \\
\left.\left(x^{k-1} * u(x, t)\right)\right|_{x=b}=0, \\
k=\overline{1, m}, \quad j=\overline{m+1,2 m,} \\
\mathfrak{S Y}=Y_{t t}+(-1)^{m} \alpha(t) \frac{\partial}{\partial t}\left(\frac{\partial^{2 m+1} Y}{\partial x^{2 m+1}}\right)=0, \\
Y(x, 0)=\varphi_{1}(x), \quad Y_{t}(x, 0)=\varphi_{2}(x), \\
Y(0, t)=0, \quad \frac{\partial^{j} Y(b, t)}{\partial x^{j}}=0, \\
\left.\left(x^{k-1} * Y(x, t)\right)\right|_{x=b}=0, \\
k=\overline{1, m}, \quad j=\frac{m+1,2 m .}{m}
\end{gathered}
$$

The function $F$ satisfies the condition

$$
\begin{gathered}
\left|F\left(x, t, \sigma_{0}, \sigma_{1}\right)-F\left(x, t, \omega_{0}, \omega_{1}\right)\right| \\
\quad \leq d\left(\left|\sigma_{0}-\omega_{0}\right|+\left|\sigma_{1}-\omega_{1}\right|\right),
\end{gathered}
$$

for all $(x, t) \in Q=(0, b) \times(0, T)$.

According to Theorem 5, problem (43) has a unique solution $Y$ depending continuously on $\varphi_{1}(x) \in H(0, b)$, $\varphi_{2}(x) \in H(0, b)$. It remains to prove that problem (40) has a unique weak solution.

Consider the inner product

$$
\begin{aligned}
\left(\mathscr{L} y, \frac{x^{2 m-1} * v}{(2 m-1) !}\right)_{L^{2}(\mathrm{Q})} \\
=\left(y_{t t}, \frac{x^{2 m-1} * v}{(2 m-1) !}\right)_{L^{2}(\mathrm{Q})} \\
\quad+(-1)^{m} \alpha(t)\left(\frac{\partial^{2 m+1} y_{t}}{\partial x^{2 m+1}}, \frac{x^{2 m-1} * v}{(2 m-1) !}\right)_{L^{2}(\mathrm{Q})}
\end{aligned}
$$

with $v, y \in C^{2}(Q)$, such that $y(x, 0)=0, y_{t}(x, 0)=0$, $y(0, t)=0, v(x, T)=0, v_{t}(x, T)=0, v(b, t)=0, x^{k-1} *$ $\left.y(x, t)\right|_{x=b}=0, \quad k=\overline{1, m},\left.x^{k-1} * v(x, t)\right|_{x=b}=0$, and $k=$ $\overline{0,2 m-1}$.
By using the above conditions on $v$ and $y$, we can write (45) in the form of

$$
\begin{aligned}
& -\left(\frac{x^{2 m-1} * v_{t}}{(2 m-1) !}, y_{t}\right)_{L^{2}(\mathrm{Q})}+(-1)^{m+1} \alpha(t)\left(y_{t}, v_{x}\right)_{L^{2}(\mathrm{Q})} \\
& =\left(\mathscr{L} y, \frac{x^{2 m-1} * v}{(2 m-1) !}\right)_{L^{2}(\mathrm{Q})} .
\end{aligned}
$$

On the other hand, we have

$$
\left(\mathscr{L} y, \frac{x^{2 m-1} * v}{(2 m-1) !}\right)_{L^{2}(\mathrm{Q})}=\left(\frac{x^{2 m-1} * \mathscr{L} y}{(2 m-1) !}, v\right)_{L^{2}(\mathrm{Q})} .
$$

It follows from (46) and (47) that

$$
\Lambda(v, y)=\left(\frac{x^{2 m-1} * \mathscr{L} y}{(2 m-1) !}, v\right)_{L^{2}(Q)}
$$

where

$$
\begin{aligned}
\Lambda(v, y)= & -\left(\frac{x^{2 m-1} * y_{t}}{(2 m-1) !}, v_{t}\right)_{L^{2}(\mathrm{Q})} \\
& +(-1)^{m+1} \alpha(t)\left(y_{t}, v_{x}\right)_{L^{2}(\mathrm{Q})} .
\end{aligned}
$$

Definition 8. One calls a function $y \in H^{1}\left(0, T ; L^{2}(0, b)\right)$ a weak solution of problem (40) if (48) and conditions $\partial^{j} y(b, t) / \partial x^{j}=0, j=\overline{m+1,2 m}$, are satisfied.

One now considers the following iterated problems:

$$
\begin{gathered}
\mathfrak{Q} y=y_{t t}^{(n)}+(-1)^{m} \alpha(t) \frac{\partial}{\partial t}\left(\frac{\partial^{2 m+1} y^{(n)}}{\partial x^{2 m+1}}\right) \\
=F\left(x, t, \frac{x^{m-1} * y^{(n-1)}}{(m-1) !}, \frac{x^{m-1} * y_{t}^{(n-1)}}{(m-1) !}\right), \\
y^{(n)}(x, 0)=0, \quad y_{t}^{(n)}(x, 0)=0, \\
y^{(n)}(0, t)=0, \quad \frac{\partial^{j} y^{(n)}(b, t)}{\partial x^{j}}=0, \\
\left.\left(x^{k-1} * y^{(n)}(x, t)\right)\right|_{x=b}=0, \\
k=\overline{1, m}, \quad j=\overline{m+1,2 m .}
\end{gathered}
$$


Theorem 5 asserts that each problem (50) admits a unique solution $y^{(n)}(x, t)$. By setting $Z^{(n)}(x, t)=y^{(n+1)}(x, t)-$ $y^{(n)}(x, t)$, one gets the following mixed iterated problem:

$$
\begin{gathered}
\mathcal{L} Z^{(n)}=Z_{t t}^{(n)}+(-1)^{m} \alpha(t) \frac{\partial}{\partial t}\left(\frac{\partial^{2 m+1} Z^{(n)}}{\partial x^{2 m+1}}\right) \\
=P^{(n-1)}(x, t), \\
Z^{(n)}(x, 0)=0, \quad Z_{t}^{(n)}(x, 0)=0, \\
Z^{(n)}(0, t)=0, \quad \frac{\partial^{j} Z^{(n)}(b, t)}{\partial x^{j}}=0, \\
\left.\left(x^{k-1} * Z^{(n)}(x, t)\right)\right|_{x=b}=0, \\
k=\overline{1, m}, \quad j=\overline{m+1,2 m},
\end{gathered}
$$

where

$$
\begin{aligned}
P^{(n-1)}(x, t)= & F\left(x, t, \frac{x^{m-1} * y^{(n)}}{(m-1) !}, \frac{x^{m-1} * y_{t}^{(n)}}{(m-1) !}\right) \\
& -F\left(x, t, \frac{x^{m-1} * y^{(n-1)}}{(m-1) !}, \frac{x^{m-1} * y_{t}^{(n-1)}}{(m-1) !}\right) .
\end{aligned}
$$

Theorem 9. Assume that condition (44) holds then there exists a positive constant $\lambda$ such that the solution $Z^{(n)}$ of problem (51) satisfies the inequality

$$
\left\|Z^{(n)}\right\|_{H^{1}\left(0, T ; L^{2}(0, b)\right)} \leq \lambda\left\|Z^{(n-1)}\right\|_{H^{1}\left(0, T ; L^{2}(0, b)\right)},
$$

where $\lambda=d \sqrt{T}\left(b^{m} / 2^{m / 2}\right) e^{T}$.

Proof. By considering the scalar product in $L^{2}\left(0, \tau ; L^{2}(0, b)\right)$, $0 \leq \tau \leq T$ of the partial differential equation in (51) and the intgrodifferential operator $\wp Z^{(n)}=Z_{t}^{(n)}$ and by using initial and boundary conditions in (51), we obtain

$$
\begin{aligned}
& \frac{1}{2} \int_{0}^{b}\left(Z_{t}^{(n)}(x, \tau)\right)^{2} d x+\int_{Q_{\tau}} \alpha(t)\left(\frac{\partial^{m} Z^{(n)}}{\partial x^{m}}\right)^{2} d x d t \\
& \quad=\left(P^{(n-1)}, Z_{t}^{(n)}\right)_{L^{2}\left(0, \tau ; L^{2}(0, b)\right)}
\end{aligned}
$$

It is easy to show the elementary inequality

$$
\begin{aligned}
\int_{0}^{b} & \left(Z^{(n)}(x, \tau)\right)^{2} d x \\
\quad \leq & \int_{0}^{b}\left(Z^{(n)}(x, 0)\right)^{2} d x+\int_{Q_{\tau}}\left(Z^{(n)}\right)^{2} d x d t \\
\quad & \quad \int_{Q_{\tau}}\left(Z_{t}^{(n)}\right)^{2} d x d t \\
= & \int_{Q_{\tau}}\left(Z^{(n)}\right)^{2} d x d t+\int_{Q_{\tau}}\left(Z_{t}^{(n)}\right)^{2} d x d t .
\end{aligned}
$$

Combination of (54) and (55) after discarding the second term on the left-hand side of (54) and using Cauchy inequality lead to

$$
\begin{aligned}
& \left\|Z^{(n)}(x, \tau)\right\|_{L^{2}(0, b)}^{2}+\left\|Z_{t}^{(n)}(x, \tau)\right\|_{L^{2}(0, b)}^{2} \\
& \quad \leq\left\|Z^{(n)}\right\|_{L^{2}\left(\mathrm{Q}_{\tau}\right)}^{2}+2\left\|Z_{t}^{(n)}\right\|_{L^{2}\left(\mathrm{Q}_{\tau}\right)}^{2}+\left\|P^{(n-1)}\right\|_{L^{2}\left(\mathrm{Q}_{\tau}\right)}^{2} .
\end{aligned}
$$

On the other hand, we have

$$
\begin{aligned}
& \left\|P^{(n-1)}\right\|_{L^{2}\left(\mathrm{Q}_{\tau}\right)}^{2} \\
& \quad \leq d^{2}\left(\left\|\frac{x^{m-1} * Z^{(n-1)}}{(m-1) !}\right\|_{L^{2}\left(\mathrm{Q}_{\tau}\right)}^{2}+\left\|\frac{x^{m-1} * Z_{t}^{(n-1)}}{(m-1) !}\right\|_{L^{2}\left(\mathrm{Q}_{\tau}\right)}^{2}\right) \\
& \quad \leq\left(\frac{b^{2}}{2}\right)^{m} d^{2}\left(\left\|Z^{(n-1)}\right\|_{L^{2}\left(\mathrm{Q}_{\tau}\right)}^{2}+\left\|Z_{t}^{(n-1)}\right\|_{L^{2}\left(\mathrm{Q}_{\tau}\right)}^{2}\right) .
\end{aligned}
$$

Combining inequalities (56) and (57) and using (11), we obtain

$$
\begin{aligned}
& \left\|Z^{(n)}(x, \tau)\right\|_{L^{2}(0, b)}^{2}+\left\|Z_{t}^{(n)}(x, \tau)\right\|_{L^{2}(0, b)}^{2} \\
& \leq 2\left[\left\|Z^{(n)}\right\|_{L^{2}\left(Q_{\tau}\right)}^{2}+\left\|Z_{t}^{(n)}\right\|_{L^{2}\left(Q_{\tau}\right)}^{2}\right] \\
& \quad+d^{2} \frac{b^{2 m}}{2^{m}}\left(\left\|Z^{(n-1)}\right\|_{L^{2}\left(Q_{\tau}\right)}^{2}+\left\|Z_{t}^{(n-1)}\right\|_{L^{2}\left(Q_{\tau}\right)}^{2}\right) .
\end{aligned}
$$

By applying Gronwall's lemma (see [22]) to inequality (58), we have

$$
\begin{aligned}
& \left\|Z^{(n)}(x, \tau)\right\|_{L^{2}(0, b)}^{2}+\left\|Z_{t}^{(n)}(x, \tau)\right\|_{L^{2}(0, b)}^{2} \\
& \quad \leq d^{2} \frac{b^{2 m}}{2^{m}} e^{2 \tau}\left(\left\|Z^{(n-1)}\right\|_{L^{2}\left(Q_{\tau}\right)}^{2}+\left\|Z_{t}^{(n-1)}\right\|_{L^{2}\left(Q_{\tau}\right)}^{2}\right) .
\end{aligned}
$$

Integration of both sides of (59) with respect to $\tau$ over $[0, T]$, yields

$$
\frac{\left\|Z^{(n)}(x, \tau)\right\|_{H^{1}\left(0, T ; L^{2}(0, b)\right)}}{\left\|Z^{(n-1)}(x, \tau)\right\|_{H^{1}\left(0, T ; L^{2}(0, b)\right)}} \leq d \sqrt{T} \frac{b^{m}}{2^{m / 2}} e^{T} .
$$

Inequality (60) implies that the series $\sum_{n=1}^{\infty} Z^{(n)}$ converges if $d \sqrt{T}\left(b^{m} / 2^{m / 2}\right) e^{T}<1$. It is obvious that the sequence $\left(y^{(n)}\right)_{n \in N}$ defined by

$$
\begin{aligned}
y^{(n)}(x, t) & =y^{(0)}(x, t)+\sum_{k=0}^{n-1} Z^{(k)} \\
& =y^{(0)}(x, t)+\sum_{k=0}^{n-1}\left(y^{(k+1)}-y^{(k)}\right), \quad n=1,2, \ldots
\end{aligned}
$$

converges to a limit function $y \in H^{1}\left(0, T ; L^{2}(0, b)\right)$ which must satisfy (48) and conditions $\partial^{j} y(b, t) / \partial x^{j}=0, j=$ $\overline{m+1,2 m}$. 
It is obvious that from the partial differential equation in (50) we have

$$
\begin{aligned}
\Lambda\left(y^{(n)}-y, v\right) \\
=\left(\frac{\partial^{2}}{\partial t^{2}} \frac{x^{2 m-1} *\left(y^{(n)}-y\right)}{(2 m-1) !}, v\right)_{L^{2}(\mathrm{Q})} \\
\quad+(-1)^{m} \\
\quad \times\left(\alpha(t) \frac{x^{2 m-1} *\left(\left(\partial^{2 m+1} / \partial x^{2 m+1}\right)\left(y_{t}^{(n)}-y_{t}\right)\right)}{(2 m-1) !}, v\right)_{L^{2}(\mathrm{Q})}
\end{aligned}
$$

and we also have

$$
\begin{aligned}
& \Lambda\left(y^{(n)}, v\right) \\
& =\left(\left(x^{2 m-1} * F\left(x, t, \frac{x^{m-1} * y^{(n-1)}}{(m-1) !}, \frac{x^{m-1} * y_{t}^{(n-1)}}{(m-1) !}\right)\right)\right. \\
& \left.\quad \times((2 m-1) !)^{-1}, v\right)_{L^{2}(\mathrm{Q})} .
\end{aligned}
$$

Equality (63) gives

$$
\begin{aligned}
\Lambda(y, v)+ & \Lambda\left(y^{(n)}-y, v\right) \\
=( & \left(x^{2 m-1} * F\left(x, t, \frac{x^{m-1} * y}{(m-1) !}, \frac{x^{m-1} * y_{t}}{(m-1) !}\right)\right) \\
& \left.\times((2 m-1) !)^{-1}, v\right)_{L^{2}(\mathrm{Q})} \\
+ & \left(\left(x^{2 m-1} * F\left(x, t, \frac{x^{m-1} * y^{(n-1)}}{(m-1) !}, \frac{x^{m-1} * y_{t}^{(n-1)}}{(m-1) !}\right)\right)\right. \\
& \times((2 m-1) !)^{-1} \\
& -\left(x^{2 m-1} * F\left(x, t, \frac{x^{m-1} * y}{(m-1) !}, \frac{x^{m-1} * y_{t}}{(m-1) !}\right)\right) \\
& \left.\times((2 m-1) !)^{-1}, v\right)_{L^{2}(\mathrm{Q})}
\end{aligned}
$$

By using conditions on $v$, evaluation of the right-hand side of (62) gives

$$
\begin{gathered}
\left(\frac{\partial^{2}}{\partial t^{2}} \frac{x^{2 m-1} *\left(y^{(n)}-y\right)}{(2 m-1) !}, v\right)_{L^{2}(Q)} \\
\quad=-\left(\frac{x^{2 m-1} *\left(y_{t}^{(n)}-y_{t}\right)}{(2 m-1) !}, v_{t}\right)_{L^{2}(\mathrm{Q})},
\end{gathered}
$$

$$
\begin{aligned}
& (-1)^{m}\left(\alpha(t) \frac{x^{2 m-1} *\left(\left(\partial^{2 m+1} / \partial x^{2 m+1}\right)\left(y_{t}^{(n)}-y_{t}\right)\right)}{(2 m-1) !}, v\right)_{L^{2}(\mathrm{Q})} \\
& =(-1)^{m+1}\left(\alpha(t)\left(y_{t}^{(n)}-y_{t}\right), v_{x}\right)_{L^{2}(\mathrm{Q})} .
\end{aligned}
$$

Combination of (62) and (65) leads to

$$
\begin{aligned}
\Lambda\left(y^{(n)}-y, v\right)= & -\left(\frac{x^{2 m-1} *\left(y_{t}^{(n)}-y_{t}\right)}{(2 m-1) !}, v_{t}\right)_{L^{2}(\mathrm{Q})} \\
& +(-1)^{m+1}\left(\alpha(t)\left(y_{t}^{(n)}-y_{t}\right), v_{x}\right)_{L^{2}(\mathrm{Q})}
\end{aligned}
$$

Application of Cauchy Shwartz to the two terms of the righthand side of (66) gives

$$
\begin{aligned}
& -\left(\frac{x^{2 m-1} *\left(y_{t}^{(n)}-y_{t}\right)}{(2 m-1) !}, v_{t}\right)_{L^{2}(\mathrm{Q})} \\
& \leq\left\|\frac{x^{2 m-1} *\left(y_{t}^{(n)}-y_{t}\right)}{(2 m-1) !}\right\|_{L^{2}(\mathrm{Q})} \cdot\left\|v_{t}\right\|_{L^{2}(\mathrm{Q})} \\
& \quad \leq \frac{b^{4 m}}{2^{2 m}}\left\|\left(y_{t}^{(n)}-y_{t}\right)\right\|_{L^{2}(\mathrm{Q})} \cdot\left\|v_{t}\right\|_{L^{2}(\mathrm{Q})} \\
& \quad \leq \frac{b^{4 m}}{2^{2 m}}\left\|y^{(n)}-y\right\|_{H^{1}\left(0, T ; L^{2}(0, b)\right)} \cdot\left\|v_{t}\right\|_{L^{2}(\mathrm{Q})}, \\
& (-1)^{m+1}\left(\alpha(t)\left(y_{t}^{(n)}-y_{t}\right), v_{x}\right)_{L^{2}(\mathrm{Q})} \\
& \quad \leq c_{1}\left\|\left(y_{t}^{(n)}-y_{t}\right)\right\|_{L^{2}(\mathrm{Q})} \cdot\left\|v_{x}\right\|_{L^{2}(\mathrm{Q})} \\
& \quad \leq c_{1}\left\|y^{(n)}-y\right\|_{H^{1}\left(0, T ; L^{2}(0, b)\right)} \cdot\left\|v_{x}\right\|_{L^{2}(\mathrm{Q})} .
\end{aligned}
$$

It follows from (66)-(67) that

$$
\begin{aligned}
& \Lambda\left(y^{(n)}-y, v\right) \\
& \leq \max \left(\frac{b^{4 m}}{2^{2 m}}, c_{1}\right)\left\|y^{(n)}-y\right\|_{H^{1}\left(0, T ; L^{2}(0, b)\right)} \\
& \cdot\left(\left\|v_{x}\right\|_{L^{2}(\mathrm{Q})}+\left\|v_{t}\right\|_{L^{2}(\mathrm{Q})}\right) .
\end{aligned}
$$

On the other hand we have

$$
\begin{aligned}
& \left(\left(x^{2 m-1} * F\left(x, t, \frac{x^{m-1} * y^{(n-1)}}{(m-1) !}, \frac{x^{m-1} * y_{t}^{(n-1)}}{(m-1) !}\right)\right)\right. \\
& \quad \times((2 m-1) !)^{-1} \\
& -\left(x^{2 m-1} * F\left(x, t, \frac{x^{m-1} * y}{(m-1) !}, \frac{x^{m-1} * y_{t}}{(m-1) !}\right)\right)
\end{aligned}
$$




$$
\begin{aligned}
& \left.\times((2 m-1) !)^{-1}, v\right)_{L^{2}(\mathrm{Q})} \\
\leq & \left(\frac{b^{4 m}}{2^{2 m}}\right) d\|v\|_{L^{2}(\mathrm{Q})} \\
& \times\left\{\left\|\frac{x^{m-1} *\left(y^{(n)}-y\right)}{(m-1) !}\right\|_{H^{1}\left(0, T ; L^{2}(0, b)\right)}\right\} \\
\leq & \left(\frac{b^{6 m+1}}{2^{3 m}}\right) d\|v\|_{L^{2}(Q)} \\
& \times\left\{\left\|y^{(n)}-y\right\|_{H^{1}\left(0, T ; L^{2}(0, b)\right)}\right\} .
\end{aligned}
$$

Now taking into account inequalities (68) and (69) and passing to limit inequality (64) as $n \rightarrow \infty$, we obtain

$$
\begin{aligned}
\Lambda(y, v)= & \left(\left(x^{2 m-1} * F\left(x, t, \frac{x^{m-1} * y}{(m-1) !}, \frac{x^{m-1} * y_{t}}{(m-1) !}\right)\right)\right. \\
& \left.\times((2 m-1) !)^{-1}, v\right)_{L^{2}(Q)}
\end{aligned}
$$

which is exactly inequality (48). Now since $y \in$ $H^{1}\left(0, T ; L^{2}(0, b)\right)$, then $\int_{0}^{t}\left(\partial^{j} y(b, t) / \partial x^{j}\right) d s \in C(\bar{Q})$, and we conclude that $\partial^{j} y(b, t) / \partial x^{j}=0, j=\overline{m+1,2 m}$, almost everywhere.

We now prove the uniqueness of solution of problem (40).

Theorem 10. Assume that condition (44) is fulfilled, then the initial boundary value problem (40) admits a unique solution.

Proof. Suppose that $S_{1}, S_{2} \in H^{1}\left(0, T ; L^{2}(0, b)\right)$ are two solutions of problem (40), then $S=S_{1}-S_{2} \in H^{1}\left(0, T ; L^{2}(0, b)\right)$ and satisfies

$$
\begin{gathered}
S_{t t}+(-1)^{m} \alpha(t) \frac{\partial}{\partial t}\left(\frac{\partial^{2 m+1} S}{\partial x^{2 m+1}}\right)=H(x, t), \\
S(x, 0)=0, \quad S_{t}(x, 0)=0 \\
S(0, t)=0, \quad \frac{\partial^{j} S(b, t)}{\partial x^{j}}=0 \\
\left.\left(x^{k-1} * S(x, t)\right)\right|_{x=b}=0 \\
k=\overline{1, m}, \quad j=\overline{m+1,2 m}
\end{gathered}
$$

where

$$
\begin{aligned}
H(x, t)= & F\left(x, t, \frac{x^{m-1} * S_{1}}{(m-1) !}, \frac{x^{m-1} * \partial S_{1} / \partial t}{(m-1) !}\right) \\
& -F\left(x, t, \frac{x^{m-1} * S_{2}}{(m-1) !}, \frac{x^{m-1} * \partial S_{2} / \partial t}{(m-1) !}\right) .
\end{aligned}
$$

As we have proceeded in the proof of Theorem 9, we consider the scalar product in $L^{2}\left(0, \tau ; L^{2}(0, b)\right)$ of the differential equation in (71) and the operator $\wp S=\partial S / \partial t$, we obtain

$$
\|S\|_{H^{1}\left(0, T ; L^{2}(0, b)\right)} \leq \lambda\|S\|_{H^{1}\left(0, T ; L^{2}(0, b)\right)}
$$

where $\lambda=d \sqrt{T}\left(b^{m} / 2^{m / 2}\right) e^{T}$.

Since it is assumed that $\lambda<1$, then it follows that $S=S_{1}-$ $S_{2}=0$. Therefore $S_{1}=S_{2}$. Hence the uniqueness of solution of problem (40) is in $H^{1}\left(0, T ; L^{2}(0, b)\right)$.

\section{Appendices}

\section{A.}

Proof of Corollary 1. We have

$$
\begin{aligned}
& \left(\frac{x^{m-1} * u(x, t)}{(m-1) !}\right)^{2} \\
& \quad=\left(\int_{0}^{x} \frac{\xi^{m-2} * u(\xi, t)}{(m-2) !} d \xi\right)^{2} \\
& \quad \leq\left(\int_{0}^{x} d \xi\right)\left(\int_{0}^{x}\left(\frac{\xi^{m-2} * u(\xi, t)}{(m-2) !}\right)^{2} d \xi\right) \\
& \quad \leq x \int_{0}^{x}\left(\frac{\xi^{m-2} * u(\xi, t)}{(m-2) !}\right)^{2} d \xi \\
& \quad \leq x \int_{0}^{b}\left(\frac{\xi^{m-2} * u(\xi, t)}{(m-2) !}\right)^{2} d \xi .
\end{aligned}
$$

Consequently,

$$
\begin{aligned}
\|u\|_{H(0, b)}^{2} & \leq \int_{0}^{b}\left(\frac{\xi^{m-2} * u(\xi, t)}{(m-2) !}\right)^{2} d x \cdot \int_{0}^{b} x d x \\
& \leq \frac{b^{2}}{2}\left\|\int_{0}^{x} \frac{(x-z)^{m-2} u(z, t)}{(m-2) !} d z\right\|_{L^{2}(0, b)}^{2} .
\end{aligned}
$$

Proof of Theorem 3. We consider the scalar product in $L^{2}\left(Q^{\tau}\right)$ of the differential equation in problem (17) and the integrodifferential operator

$$
M u=2(-1)^{m} \frac{x^{2 m-1} * u_{t}(x, t)}{(2 m-1) !},
$$

where $Q^{\tau}=(0, b) \times(0, \tau)$ and $0 \leq \tau \leq T$, we obtain

$$
\begin{aligned}
& (\mathbb{\Omega} u, M u)_{L^{2}\left(Q^{\tau}\right)} \\
& =2(-1)^{m}\left(u_{t t}, \frac{x^{2 m-1} * u_{t}(x, t)}{(2 m-1) !}\right)_{L^{2}\left(Q^{\tau}\right)} \\
& \quad+2\left(\alpha(t) \frac{\partial^{2 m+1} u_{t}}{\partial x^{2 m+1}}, \frac{x^{2 m-1} * u_{t}(x, t)}{(2 m-1) !}\right)_{L^{2}\left(Q^{\tau}\right)} .
\end{aligned}
$$


We separately consider the integrals in the right-hand side of (A.4) and we integrate by parts and taking into account boundary and initial conditions in (17), we obtain

$$
\begin{gathered}
2(-1)^{m}\left(u_{t t}, \frac{x^{2 m-1} * u_{t}(x, t)}{(2 m-1) !}\right)_{L^{2}\left(Q^{\tau}\right)} \\
=\int_{0}^{b}\left(\frac{x^{m-1} * u_{t}(x, \tau)}{(m-1) !}\right)^{2} d x \\
-\int_{0}^{b}\left(\frac{x^{m-1} * \varphi_{2}}{(m-1) !}\right)^{2} d x, \\
2\left(\alpha(t) \frac{\partial^{2 m+1} u_{t}}{\partial x^{2 m+1}}, \frac{x^{2 m-1} * u_{t}(x, t)}{(2 m-1) !}\right)_{L^{2}\left(Q^{\tau}\right)} \\
=\int_{0}^{\tau} \alpha(t) u_{t}^{2}(b, t) d t .
\end{gathered}
$$

Substitution of (A.5) into (A.4) yields

$$
\begin{gathered}
\int_{0}^{\tau} \alpha(t) u_{t}^{2}(b, 0) d t+\int_{0}^{b}\left(\frac{x^{m-1} * u_{t}(x, \tau)}{(m-1) !}\right)^{2} d x \\
=(\mathfrak{Q} u, M u)_{L^{2}\left(Q^{\tau}\right)}+\int_{0}^{b}\left(\frac{x^{m-1} * \varphi_{2}}{(m-1) !}\right)^{2} d x .
\end{gathered}
$$

By Corollary 1, we have

$$
\begin{aligned}
& (\boldsymbol{\Omega} u, M u)_{L^{2}\left(Q^{\tau}\right)} \\
& \quad \leq\|f\|_{L^{2}\left(Q^{\tau}\right)}^{2}+\left(\frac{b^{2}}{2}\right)^{m}\left\|u_{t}\right\|_{L^{2}(0, \tau ; H(0, b))}^{2} .
\end{aligned}
$$

If we discard the first term in (A.6), and by using (A.7), we obtain

$$
\begin{aligned}
\left\|u_{t}(x, \tau)\right\|_{H(0, b)}^{2} \leq & \|f\|_{L^{2}\left(Q^{\tau}\right)}^{2}+\left\|\varphi_{2}\right\|_{H(0, b)}^{2} \\
& +\left(\frac{b^{2}}{2}\right)^{m}\left\|u_{t}\right\|_{L^{2}(0, \tau ; H(0, b))}^{2} .
\end{aligned}
$$

By virtue of the elementary inequality

$$
\begin{aligned}
& \|u(x, \tau)\|_{H(0, b)}^{2} \\
& \quad \leq\left\|\varphi_{1}\right\|_{H(0, b)}^{2}+\|u\|_{L^{2}(0, \tau ; H(0, b))}^{2}+\left\|u_{t}\right\|_{L^{2}(0, \tau ; H(0, b))}^{2},
\end{aligned}
$$

and (A.8), we have

$$
\begin{aligned}
& \|u(x, \tau)\|_{H(0, b)}^{2}+\left\|u_{t}(x, \tau)\right\|_{H(0, b)}^{2} \\
& \leq\|f\|_{L^{2}\left(Q^{\tau}\right)}^{2}+\left\|\varphi_{1}\right\|_{B_{2}^{m}(0, b)}^{2}+\left\|\varphi_{2}\right\|_{B_{2}^{m}(0, b)}^{2} \\
& \quad+c_{5}\left(\|u\|_{L^{2}(0, \tau ; H(0, b))}^{2}+\left\|u_{t}\right\|_{L^{2}(0, \tau ; H(0, b))}^{2}\right),
\end{aligned}
$$

where

$$
c_{5}=1+\left(\frac{b^{2}}{2}\right)^{m} .
$$

Let

$$
\begin{aligned}
& h_{1}(t)=0, \\
& h_{2}(t)=\|u(x, \tau)\|_{H(0, b)}^{2}+\left\|u_{t}(x, \tau)\right\|_{H(0, b)}^{2}, \\
& h_{3}(t)=\|f\|_{L^{2}\left(Q^{\tau}\right)}^{2}+\left\|\varphi_{1}\right\|_{H(0, b)}^{2}+\left\|\varphi_{2}\right\|_{H(0, b)}^{2},
\end{aligned}
$$

then it follows from Gronwall's lemma (see [3]) that

$$
\begin{aligned}
& \|u(x, \tau)\|_{H(0, b)}^{2}+\left\|u_{t}(x, \tau)\right\|_{H(0, b)}^{2} \\
& \quad \leq \exp \left(c_{5} \tau\right) \cdot\left(\|f\|_{L^{2}\left(Q^{\tau}\right)}^{2}+\left\|\varphi_{1}\right\|_{H(0, b)}^{2}+\left\|\varphi_{2}\right\|_{H(0, b)}^{2}\right) \\
& \quad \leq \exp \left(c_{5} T\right) \cdot\left(\|f\|_{L^{2}(\mathrm{Q})}^{2}+\left\|\varphi_{1}\right\|_{H(0, b)}^{2}+\left\|\varphi_{2}\right\|_{H(0, b)}^{2}\right) .
\end{aligned}
$$

The right-hand side in (A.13) is independent of $\tau$, hence replacing the left-hand side by its upper bound with respect to $\tau$ over $[0, T]$, we obtain (18), with $M=\exp \left(c_{5} T\right)$, and Theorem 3 follows.

\section{B.}

Proof of Lemma 7. We have

$$
\begin{aligned}
\Psi(x, t)= & \alpha^{\prime \prime}(t) \frac{x^{2 m-1} * u_{t}(x, t)}{(2 m-1) !} \\
& +2 \alpha^{\prime}(t) \frac{x^{2 m-1} * u_{t t}(x, t)}{(2 m-1) !} \\
& +\alpha(t) \frac{\partial}{\partial t}\left(\frac{x^{2 m-1} * u_{t}(x, t)}{(2 m-1) !}\right) .
\end{aligned}
$$

It is obvious that the first and second terms on the right-side of (B.1) are in $L^{2}(Q)$. To prove that the term $\alpha(t)(\partial / \partial t)\left(\left(x^{2 m-1} * u_{t}(x, t)\right) /(2 m-1) !\right) \in L^{2}(Q)$, we use the $t$-averaging operators $\rho_{\varepsilon}$ of the form

$$
\left(\rho_{\varepsilon} v\right)(x, t)=\frac{1}{\varepsilon} \int_{-\infty}^{+\infty} W\left(\frac{s-t}{\varepsilon}\right) v(x, s) d s,
$$

where $W \in C_{0}^{\infty}(0, T), W(t) \geq 0, \int_{I R} W(t) d t=1$.

If we apply the operators $\rho_{\varepsilon}$ and $\partial / \partial t$ to (23), we obtain

$$
\begin{aligned}
& \alpha(t) \frac{\partial}{\partial t} \rho_{\varepsilon} \frac{x^{2 m-1} * u_{t t}(x, t)}{(2 m-1) !} \\
&=-\alpha^{\prime}(t) \rho_{\varepsilon} \frac{x^{2 m-1} * u_{t t}(x, t)}{(2 m-1) !}+\frac{\partial}{\partial t} \rho_{\varepsilon} g \\
&+\frac{\partial}{\partial t}\left(\alpha(t) \rho_{\varepsilon} \frac{x^{2 m-1} * u_{t t}(x, t)}{(2 m-1) !}\right. \\
&\left.-\rho_{\varepsilon} \alpha(t) \frac{x^{2 m-1} * u_{t t}(x, t)}{(2 m-1) !}\right) .
\end{aligned}
$$


It follows from (B.3) that

$$
\begin{gathered}
c_{0}^{2}\left\|\frac{\partial}{\partial t} \rho_{\varepsilon} \frac{x^{2 m-1} * u_{t t}(x, t)}{(2 m-1) !}\right\|_{L^{2}\left(Q_{s}\right)}^{2} \\
\leq 3 c_{3}^{2}\left\|\rho_{\varepsilon} \frac{x^{2 m-1} * u_{t t}(x, t)}{(2 m-1) !}\right\|_{L^{2}\left(Q_{s}\right)}^{2}+3\left\|\frac{\partial}{\partial t} \rho_{\varepsilon} g\right\|_{L^{2}\left(Q_{s}\right)}^{2} \\
+3 \| \frac{\partial}{\partial t}\left(\alpha(t) \rho_{\varepsilon} \frac{x^{2 m-1} * u_{t t}(x, t)}{(2 m-1) !}\right. \\
\left.\quad-\rho_{\varepsilon} \alpha(t) \frac{x^{2 m-1} * u_{t t}(x, t)}{(2 m-1) !}\right) \|_{L^{2}\left(Q_{s}\right)}^{2} .
\end{gathered}
$$

Now by using the properties of the $t$-averaging operators $\rho_{\varepsilon}$, conditions ( $\left.\mathrm{H} 1\right)$ and $(\mathrm{H} 2)$, and Corollary 2, we see from (B.4) that

$$
\begin{aligned}
& \left\|\frac{x^{2 m-1} * u_{t t t}(x, t)}{(2 m-1) !}\right\|_{L^{2}\left(\mathrm{Q}_{s}\right)}^{2} \\
& \quad \leq c_{6}\left[\left\|u_{t t}\right\|_{L^{2}\left(\mathrm{Q}_{s}\right)}^{2}+\left\|\frac{\partial}{\partial t} \rho_{\varepsilon} g\right\|_{L^{2}\left(\mathrm{Q}_{s}\right)}^{2}\right],
\end{aligned}
$$

where

$$
c_{6}=\frac{3}{c_{0}^{2}} \max \left(1, \frac{c_{3}^{2} b^{4 m}}{2^{2 m}}\right) .
$$

Since $\rho_{\varepsilon} h \rightarrow h$ in $L^{2}(Q)$ as $\varepsilon \rightarrow 0$ and the norm of $\left(x^{2 m-1} *\right.$ $\left.u_{x \times}(x, t)\right) /(2 m-1)$ ! in $L^{2}(Q)$ is bounded, we conclude that $\alpha(t)(\partial / \partial t)\left(\left(x^{2 m-1} * u_{t t t}(x, t)\right) /(2 m-1) !\right) \in L^{2}(Q)$.

\section{Conflict of Interests}

The author declares that there is no conflict of interests regarding the publication of this paper.

\section{Acknowledgment}

The author would like to extend his sincere appreciation to the Deanship of Scientific Research at King Saud University for its funding of this research through the Research Group Project no. RGP-VPP-117.

\section{References}

[1] I. G. Petrovski, "Über das cauchyshe problem für system von linearen partiallen differentialgeinchungen in Gebeit der nichtanalystischen funktionen," Bulletin de l'Université d'État de Moscow, vol. 7, pp. 1-74, 1938.

[2] J. Leray, Lectures on Hyperbolic Differential Equations with Variable Coeffecients, The Institute for Advanced Study, Princeton, NJ, USA, 1952.

[3] L. Garding, Cauchy's Problem for Hyperbolic Equations, Lecture Notes, University of chicago, 1957.
[4] A. A. Dezin, “Théorèmes d'existence et d'unicité de la solution pour les problèmes aux limites des équations aux dérivées partielles dans les espaces fonctionnels," Uspekhi Matematicheskikh Nauk, vol. 14, no. 3 (87), pp. 22-73, 1959.

[5] B. Carbonaro and R. Russo, "Energy inequalities and the domain of influence theorem in classical elastodynamics," Journal of Elasticity, vol. 14, no. 2, pp. 163-174, 1984.

[6] S. K. Godounov, "Intégrale d'énergie des équations hyperboliques d'après Petrovski," Commentationes Mathematicae Universitatis Carolinae, vol. 26, no. 1, pp. 41-74, 1985.

[7] S. K. Godunov and A. M. Blokhin, "Energy integrals in the theory of shock wave stability," in Nolinear Deformation Waves, IUTAM Symposium, Tallin, Estonia, pp. 18-29, Springer, 1983.

[8] V. I. Korzyuk, "An energy inequality for a boundary value problem for a third-order hyperbolic equation with a wave operator," Differentsial'nye Uravneniya, vol. 27, no. 6, pp. 1014$1022,1991$.

[9] F. E. Lomovtsev, "Necessary and sufficient conditions for the unique solvability of the Cauchy problem for second-order hyperbolic differential equations with a variable domain of operator coefficients," Differentsial'nye Uravneniya, vol. 28, no. 5, pp. 712-722, 1992.

[10] R. Sakamoto, "Mixed problems for hyperbolic equations. I. Energy inequalities," Journal of Mathematics of Kyoto University, vol. 10, pp. 349-373, 1970.

[11] R. Sakamoto, "Mixed problems for hyperbolic equations. II. Existence theorems with zero initial datas and energy inequalities with initial datas," Journal of Mathematics of Kyoto University, vol. 10, pp. 403-417, 1970.

[12] F. E. Lomovcev and N. I. Yurchuk, "The Cauchy problem for second order hyperbolic operator differential equations," Differentsial'nye Uravneniya, vol. 12, no. 12, pp. 2242-2250, 1976.

[13] S. A. Aldashev, "A priori estimates for the Tricomi and Darboux problems," Differentsial'nye Uravneniya, vol. 19, no. 6, pp. 985991, 1983.

[14] N. V. Kislov, "Certain operator-differential equations of mixed type," Trudy Moskovskogo Ordena Lenina Ėnergeticheskogo Instituta, vol. 146, pp. 60-69, 1972.

[15] N. V. Kislov, "Boundary value problems for operator-differential equations of mixed type," Differentsial'nye Uravneniya, vol. 19, no. 8, pp. 1427-1436, 1983.

[16] V. I. Korzyuk and V. V. Daĭnyak, "On a weak solution of a Dirichlet-type problem for a third-order linear differential equation," Differentsial'nye Uravneniya, vol. 28, no. 6, pp. 1056$1066,1992$.

[17] I. V. Suveǐka, "Mixed problems for an equation for propagation of disturbances in viscous media," Differentsial'nye Uravneniya, vol. 19, no. 2, pp. 337-347, 1983.

[18] N. V. Tsyvis and N. I. Yurchuk, "A three-point problem for third-order operator-differential equations," Differentsial'nye Uravneniya, vol. 23, no. 5, pp. 877-881, 1987.

[19] N. I. Yurchuk, "A partly characteristic boundary value problem for a certain form of partial differential equations. I," Differentsial'nye Uravneniya, vol. 4, pp. 2258-2267, 1968.

[20] A. Bouziani, "Mixed problem with boundary integral conditions for a certain parabolic equation," Journal of Applied Mathematics and Stochastic Analysis, vol. 9, no. 3, pp. 323-330, 1996.

[21] A. V. Kartynnik, "Three-point boundary value problem with an integral space-variable condition for a second order parabolic equation," Differential Equations, vol. 26, pp. 1160-1162, 1990. 
[22] A. Friedman, "Monotonic decay of solutions of parabolic equations with nonlocal boundary conditions," Quarterly of Applied Mathematics, vol. 44, no. 3, pp. 401-407, 1986.

[23] Y. Lin, Partial differential equation subject to nonlocal boundary conditions [Ph.D. thesis], Washington State University, Pullman, Wash, USA, 1988.

[24] A. Bouziani, "Solvability of nonlinear pseudoparabolic equation with a nonlocal boundary condition," Nonlinear Analysis: Theory, Methods \& Applications A: Theory and Methods, vol. 55, no. 7-8, pp. 883-904, 2003.

[25] S. Mesloub and A. Bouziani, "Problème mixte avec conditions aux limites intégrales pour une classe d'équations paraboliques bidimensionnelles," Bulletin de la Classe des Sciences, Académie Royale de Belgique, vol. 6, pp. 59-69, 1998.

[26] S. Mesloub and A. Bouziani, "Mixed problem with integral conditions for a certain class of hyperbolic equations," Journal of Applied Mathematics, vol. 1, no. 3, pp. 107-116, 2001.

[27] L. S. Pulkina, "A non-local problem with integral conditions for hyperbolic equations," Electronic Journal of Differential Equations, vol. 45, pp. 1-6, 1999.

[28] L. S. Pulkina, "On the solvability in $L_{2}$ of a nonlocal problem with integral conditions for a hyperbolic equation," Differentsial'nye Uravneniya, vol. 36, no. 2, pp. 316-318, 2000.

[29] R. J. Chegis, "Numerical solution of a heat conduction problem with an integral condition," Lithuanian Mathematical Journal, vol. 24, no. 4, pp. 209-215, 1984.

[30] G. Fairweather and J. C. López-Marcos, "Galerkin methods for a semilinear parabolic problem with nonlocal boundary conditions," Advances in Computational Mathematics, vol. 6, pp. 243-262, 1996.

[31] A. V. Goolin, N. I. Ionkin, and V. A. Morozova, "Difference schemes with nonlocal boundary conditions," Computational Methods in Applied Mathematics, vol. 1, no. 1, pp. 62-71, 2001.

[32] S. Mesloub and F. Mesloub, "On the higher dimension Boussinesq equation with nonclassical condition," Mathematical Methods in the Applied Sciences, vol. 34, no. 5, pp. 578-586, 2011. 


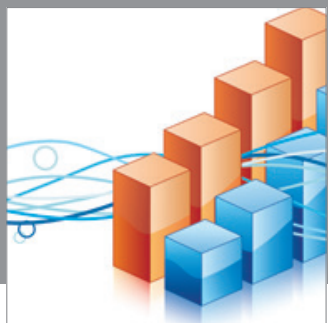

Advances in

Operations Research

mansans

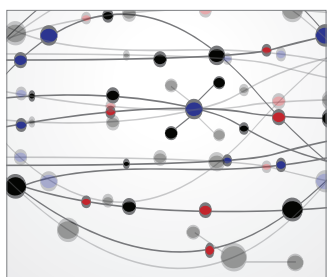

The Scientific World Journal
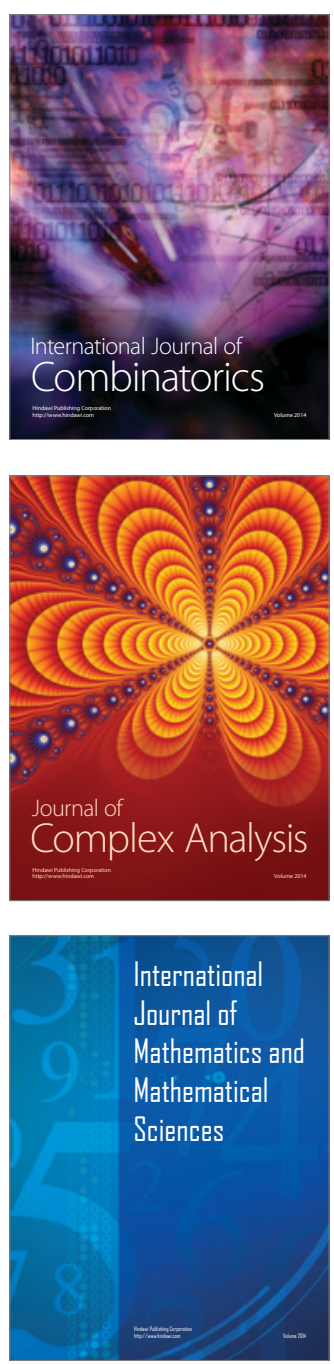
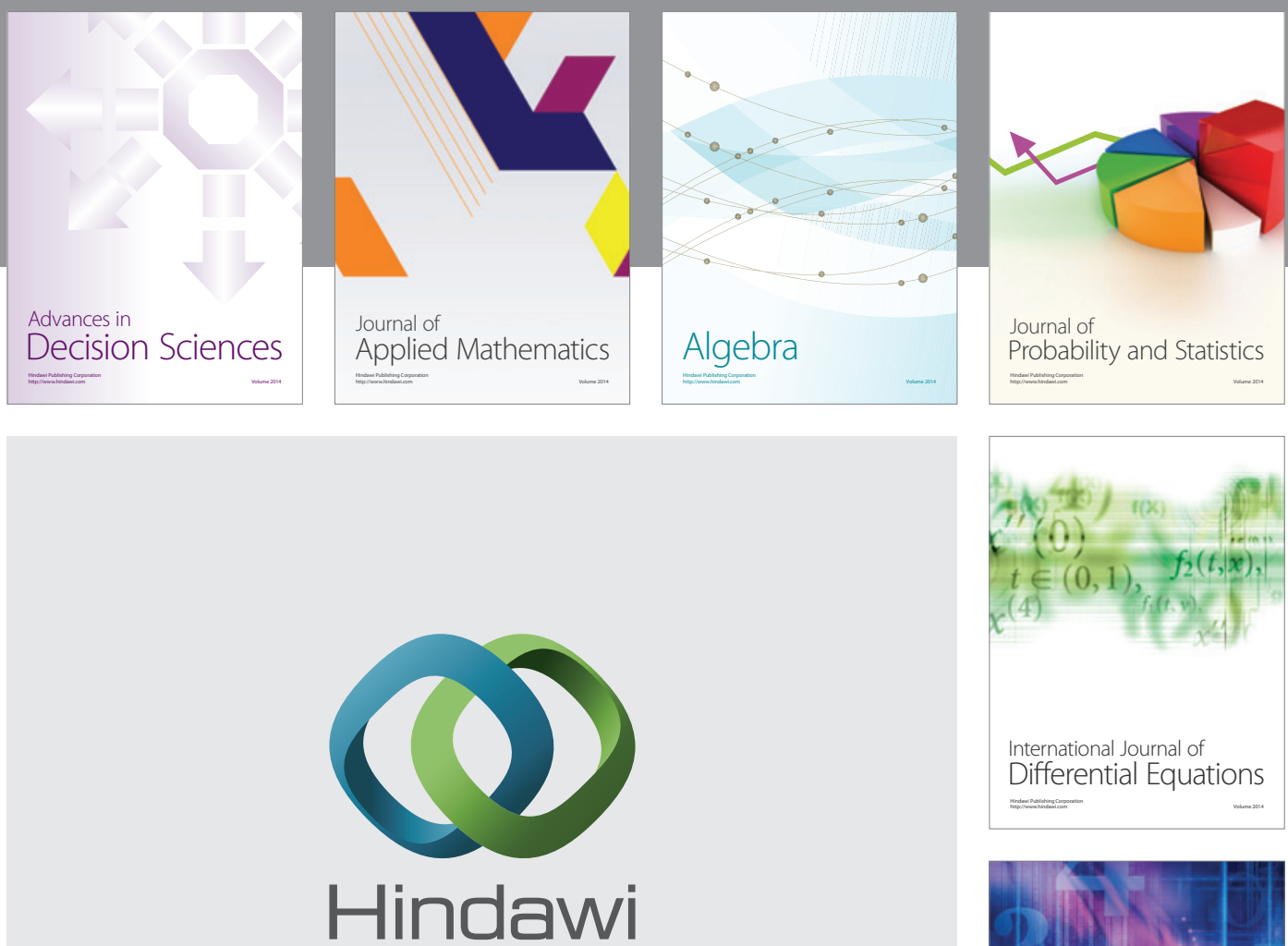

Submit your manuscripts at http://www.hindawi.com
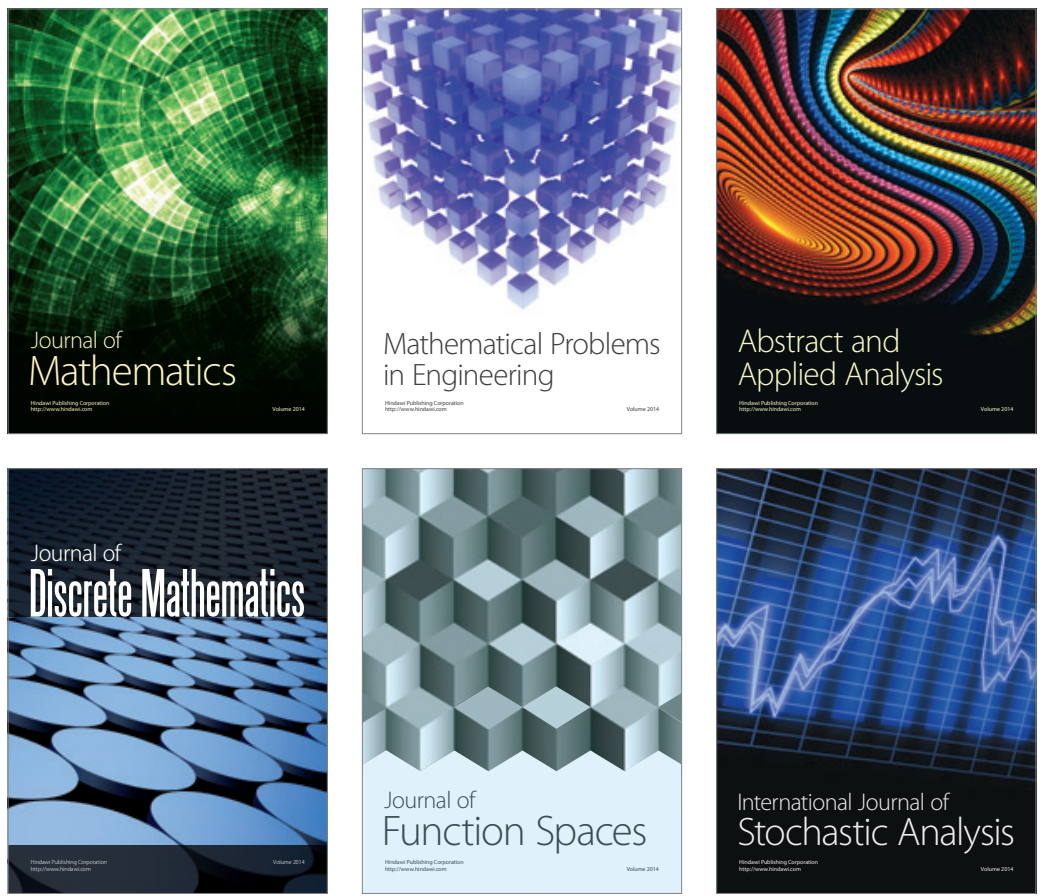

Journal of

Function Spaces

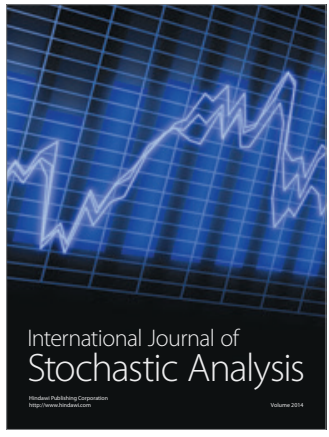

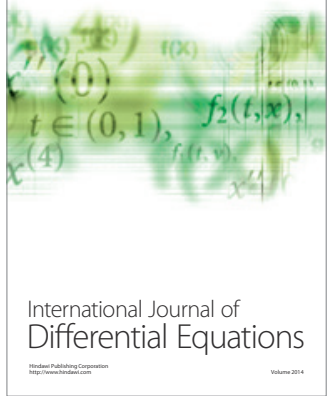
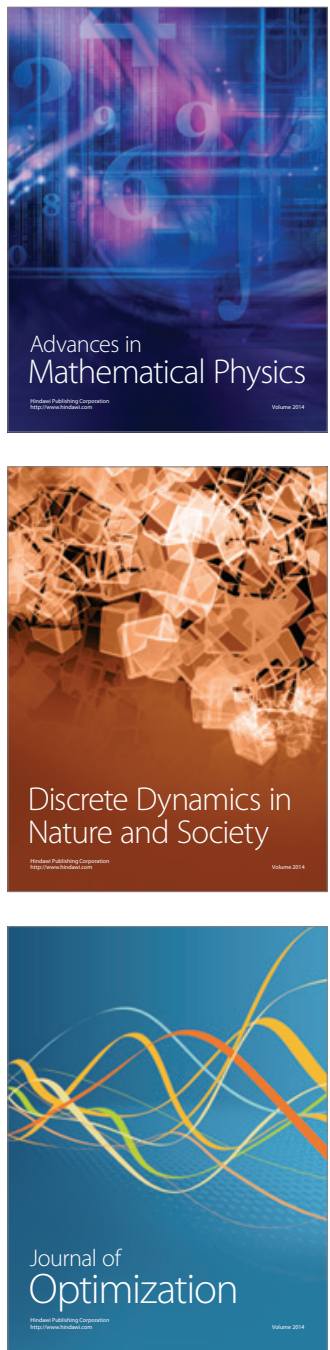